\title{
Infecciones vaginales en un Centro de Salud Familiar de la Región Metropolitana, Chile
}

\author{
Rodrigo Villaseca, Alfredo Ovalle, Fernando Amaya, Bayron Labra, Natalia Escalona, \\ Paola Lizana, M. José Montoya, Eduardo Lillo y M. Angélica Martínez
}

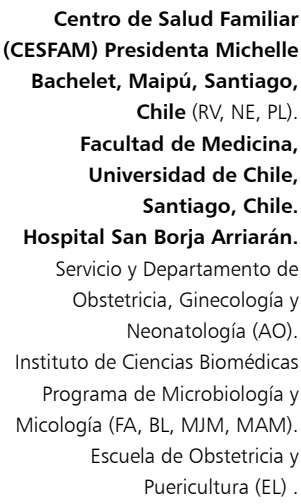

Centro de Salud Familiar (CESFAM) Presidenta Michelle Bachelet, Maipú, Santiago, Chile (RV, NE, PL) Facultad de Medicina, Universidad de Chile, Santiago, Chile. Hospital San Borja Arriarán. Servicio y Departamento de Obstetricia, Ginecología y Neonatología (AO)

Instituto de Ciencias Biomédicas

Programa de Microbiología y Micología (FA, BL, MJM, MAM).

Escuela de Obstetricia y Puericultura (EL)

Recibido: 12 de marzo de 2014 Aceptado: 9 de septiembre de 2014

No existen conflictos de interés. Financiamiento: Proyecto FONIS SA1212242

Correspondencia a: María Angélica Martínez Tagle mamartin@med.uchile.c

\section{Vaginal infections in a Family Health Clinic in the Metropolitan Region, Chile}

Background: Vaginal infections are a frequent cause for consultation, but their prevalence and etiology vary in different populations. Objectives: To determine the prevalence and etiologies of vaginal infection in women attending a family health center in the Metropolitan Region of Chile. Methods: The microbiological diagnosis was made by wet mount and Gram stain. Diagnosis of trichomoniasis was performed by wet mount, culture and polymerase chain reaction. Results: 101 women aged 15-54, not selected by signs or symptoms of vaginal infection, 46 of them pregnant were included. In 47 women (46.5\%), vaginal infections were diagnosed. An association was observed between age and frequency of vaginal infection. The proportion of infections among pregnant and non-pregnant women was similar. The most frequent infections were bacterial vaginosis $(16.8 \%)$, vulvovaginal candidiasis (11.9\%) and co-infections (6.9\%). We found 5.9\% of intermediate microbiota cases, $3 \%$ of trichomoniasis and $2 \%$ of aerobic vaginitis. Symptoms of vaginal infection had poor agreement with microbiological findings. Otherwise physical signs had good agreement with the presence of infection, but low to moderate concordance with a specific etiology. Conclusions: We found a high prevalence of vaginal infections in the study population. It is necessary to improve the definitions and criteria of microbiological diagnosis of co-infections and intermediate microbiota, for them to be diagnosed in the clinical practice. More descriptive questionnaires are recommended to enhance the usefulness of clinical examination.

Key words: Vaginal infections, vaginal microbiota, bacterial vaginosis, etiology, prevalence.

Palabras clave: Infecciones vaginales, microbiota vaginal, vaginosis bacteriana, etiología, prevalencia.

\section{Introducción}

L as infecciones vaginales constituyen un motivo frecuente de consulta en atención primaria en todo el mundo ${ }^{1,2}$. Su importancia se debe a que disminuyen la calidad de vida, favorecen el contagio de infecciones de transmisión sexual (ITS) bacterianas y virales y predisponen a enfermedad inflamatoria pélvica, parto prematuro (PP) e infección neonatal ${ }^{3-6}$. El diagnóstico clínico de estos procesos infecciosos es fundamental para establecer una terapia, pero éste se orienta fundamentalmente a reconocer las etiologías más frecuentes: vaginosis bacteriana (VB), candidiasis vulvo-vaginal $(\mathrm{CVV})$ y tricomoniasis ${ }^{7,8}$. La presentación clínica de las infecciones vaginales no es siempre característica y con frecuencia se presentan co-infecciones ${ }^{7-11}$. Por otra parte, en los últimos años se han descrito nuevas causas de infección vaginal como la vaginitis aeróbica (VA) y la microbiota intermedia (MI), cuya prevalencia es escasamente conocida ya que requieren el apoyo del laboratorio para su diagnóstico ${ }^{6,12}$. Las infecciones vaginales son generalmente el resultado de alteraciones de la microbiota vaginal, donde la microbiota normal lactobacilar es sustituida por una proliferación de otras bacterias comensales ${ }^{4-6}$. Aunque, con la excepción de la tricomoniasis las infecciones vaginales no se consideran ITS, actualmente se ha reconocido la importancia de los factores de riesgo de ITS y de algunas prácticas sexuales en su patogenia ${ }^{13,14}$. Con menor frecuencia, las molestias vaginales pueden deberse a reacciones alérgicas o irritación causada por químicos presentes en compuestos utilizados en la higiene, lubricantes, látex, entre otros ${ }^{7,8}$.

La prevalencia de las etiologías de infección vaginal varía en distintas poblaciones, por lo que los objetivos de este estudio son determinar la prevalencia y tipos de infección vaginal en mujeres sintomáticas y no sintomáticas atendidas en un CESFAM (Centro de Salud Familiar) de la Región Metropolitana (RM) de Chile.

\section{Pacientes y Métodos}

\section{Consideraciones éticas}

El estudio fue aprobado por el Comité de Ética del Servicio de Salud Metropolitano Central y financiado por el proyecto FONIS SA12I2242. Todas las pacientes firmaron un consentimiento informado. 


\section{Diseño}

Estudio descriptivo de corte transversal, efectuado entre junio y diciembre de 2013.

\section{Población estudiada}

Se enrolaron 108 mujeres de 16 a 54 años, sexualmente activas, que consultaron consecutivamente en el CESFAM Presidenta Michelle Bachelet, en la comuna de Maipú. Las muestras se tomaron, por conveniencia del laboratorio, semanalmente los jueves. Se excluyeron mujeres que recibieron antimicrobianos en el último mes.

\section{Examen clínico y recolección de muestras}

El examen clínico ginecológico y recolección de muestras de flujo vaginal fue realizado por matronas (es) del CESFAM quienes llenaron una ficha donde se anotó el motivo de consulta, la presencia de síntomas, antecedentes clínicos y los resultados del examen genital. Los síntomas fueron resumidos en prurito/irritación vulvar o secreción vaginal. Los signos fueron categorizados como inflamación (eritema/edema) y leucorrea o no especificados.

Se obtuvieron tres muestras vaginales con tórula, bajo especuloscopia. La primera fue colocada en $2 \mathrm{ml} \mathrm{de} \mathrm{NaCl}$ 9\% (suero fisiológico-SF) estéril para examen al fresco, recuento leucocitario, observación de trofozoitos móviles y RPC de Trichomonas vaginalis, detección de elementos levaduriformes, células parabasales y clue cells. Con la segunda muestra se preparó un frotis para tinción de Gram. La última muestra fue inoculada en medio de transporte Amies (COPAN) para efectuar cultivo de bacterias anaerobias facultativas, anaerobias estrictas, levaduras y T. vaginalis. Las muestras fueron conservadas y transportadas al laboratorio a temperatura ambiente dentro de las $2 \mathrm{~h}$ de obtenidas e inmediatamente procesadas.

\section{Diagnósticos}

Los diagnósticos de microbiota normal, vaginosis bacteriana y microbiota vaginal intermedia fueron efectuados utilizando el criterio de Nugent y $\operatorname{cols}^{15}$. $\mathrm{Va}$ ginitis aeróbica (VA) fue definida como la disminución sustancial o ausencia de lactobacilos y la presencia de cocáceas o bacilos grampositivos gruesos, o bacilos gramnegativos a la tinción directa, más la presencia de células parabasales y de respuesta inflamatoria definida por la presencia de leucocitos polimorfonucleares-PMN en número $>10 /$ campo de observación al fresco $(400 \mathrm{X})^{12}$.

El diagnóstico de candidiasis vulvo-vaginal (CVV) fue efectuado por la observación de levaduras, hifas o pseudohifas al examen al fresco o mediante tinción de Gram acompañado por el cultivo positivo para especies de Candida. Tricomoniasis fue diagnosticada por el hallazgo de $T$. vaginalis mediante examen al fresco o cultivo, o a la presencia de ADN del protozoo mediante $\mathrm{RPC}^{16}$.

Se consideraron infecciones vaginales al diagnóstico microbiológico de $\mathrm{VB}, \mathrm{MI}, \mathrm{VA}, \mathrm{CVV}$, tricomoniasis y de co-infecciones. Estas últimas correspondieron a la detección simultánea de VB y CVV o de VB y VA.

\section{Cultivos}

Para el cultivo de T. vaginalis las muestras fueron inoculadas en caldo Diamond modificado e incubadas a $35,5^{\circ} \mathrm{C}$, en aerobiosis por 5 días. Alícuotas del cultivo fueron observadas al microscopio con $400 \mathrm{X}$ a las 24 , 72 y $96 \mathrm{~h}$, en busca de trofozoitos móviles ${ }^{16}$. El cultivo de Candida spp. fue efectuado en agar Sabouraud (BD Difco), con incubación en aerobiosis a $35,5^{\circ} \mathrm{C}$ por 5 días. Colonias aisladas fueron subcultivadas en CHROMAgar Candida Medium ${ }^{\circledR}$ (BD Difco), con el objeto de identificar presuntivamente las especies y los cultivos mixtos. La identificación de las levaduras se efectuó mediante la determinación de tubo germinativo y microcultivo en agar maíz ${ }^{17}$. La diferenciación entre C. albicans y $C$. dubliniensis fue efectuada determinando la acidificación de la D-xilosa y de la actividad $\beta$-D-acetilglucosaminidasa (Rosco Diagnostica ${ }^{\circledR}$, Dinamarca). Ambas pruebas son positivas en C. albicans y negativas en C. dubliniensis.

\section{Reacción de polimerasa en cadena (RPC)}

El diagnóstico molecular de $T$. vaginalis fue efectuado por amplificación del ADN mediante RPC convencional con los partidores TVK3 y TVK7 y el procedimiento descrito por los autores ${ }^{18}$. La extracción del ADN fue efectuada procesando $800 \mu \mathrm{L}$ de la muestra en SF con solución de tiocianato de guanidinio -fenol-cloroformo, de acuerdo al método de Chomczynski y cols. ${ }^{19}$. Se incluyeron controles positivos (muestras positivas obtenidas de estudios anteriores) y negativos (agua) en cada corrida de RPC. Como control de la calidad de la muestra para RPC se amplificó en todas las muestras $327 \mathrm{pb}$ del gen $\beta$-globina ${ }^{16}$. Los productos de amplificación fueron analizados por electroforesis en geles de agarosa al 1,5\% teñidos con GelRed ${ }^{\mathrm{MR}}$ (Gene X-Press) y visualizados con transiluminador de luz UV.

\section{Análisis de datos}

Se utilizó el método $Z$ de dos colas para la comparación entre dos proporciones muestrales (http://www. surveystar.com/) y el test de $\chi^{2}$ para probar la asociación entre dos variables (http://www.quantpsy.org/chisq/ chisq.htm). La concordancia entre la presencia de signos de infección vaginal y el diagnóstico microbiológico fue calculada mediante el índice de $\kappa$ y valorada mediante la siguiente escala: $<0,20$, baja; 0,20-0,39, débil; 0,40-0,59, moderada; $0,60-0,79$ : buena y $0,80-1,00$, muy buena. Los intervalos de confianza [IC95\%] de las medias muestrales fueron calculados mediante el método de Agresti-Coull con ajuste de Wald. Valores de $\mathrm{p}<0,05$ fueron considerados significativos. 


\section{Resultados}

De las 108 mujeres invitadas a participar se incluyeron 101 en el estudio. Dos pacientes fueron excluidas por falta de ficha clínica y 5 por baja calidad de los frotis para efectuar la tinción de Gram. En la Tabla 1 se presentan las características de las pacientes. Cuarenta y seis participantes $(45,5 \%)$ correspondieron a mujeres embarazadas y $55(54,5 \%)$ a no embarazadas. La edad promedio de las mujeres gestantes fue 24,4 años (DE: 4,98$)$ y de las no

Tabla 1. Características de las pacientes evaluadas. Centro de Salud Familiar de Maipú, Región Metropolitana, Chile

\begin{tabular}{lrc}
\hline Característica & $\mathbf{n}$ & $\mathbf{( \% )}$ \\
$\mathrm{n}$ total & 101 & \\
$\quad$ Embarazadas & 46 & $(45,5)$ \\
$\quad$ No embarazada & 55 & $(54,5)$ \\
Edad (años), promedio (DE) & & \\
$\quad$ Embarazadas & 24,4 & $(4,98)$ \\
$\quad$ No embarazadas & 33,5 & $(10,88)$ \\
Motivo de consulta, n (\%) & & \\
$\quad$ Control prenatal & 37 & $(36,6)$ \\
$\quad$ Síntomas de infección vulvo-vaginal & 33 & $(32,7)$ \\
$\quad$ Control ginecológico & 18 & $(17,8)$ \\
$\quad$ No especificado & 13 & $(12,9)$ \\
\hline
\end{tabular}

Tabla 2. Frecuencia de microbiota normal e infecciones vaginales en mujeres embarazadas y no embarazadas. Centro de Salud Familiar de Maipú, Región Metropolitana, Chile

\begin{tabular}{lrrrrrr} 
Categorías & $\mathbf{n}$ & $\mathbf{\%}$ & Mujeres embarazadas & Mujeres no embarazadas \\
& & & $\mathbf{n}$ & $\%$ & $\mathbf{n}$ & $\%$ \\
Normal & 54 & 53,5 & 26 & 56,5 & 28 & 50,9 \\
Vaginosis bacteriana & 17 & 16,8 & 5 & 10,9 & 12 & 21,8 \\
Candidiasis vulvo-vaginal & 12 & 11,9 & 5 & 10,9 & 7 & 12,7 \\
Co-infecciones & 7 & 6,9 & 5 & 10,9 & 2 & 3,6 \\
Microbiota intermedia & 6 & 5,9 & 3 & 6,5 & 3 & 5,5 \\
Tricomoniasis & 3 & 3,0 & 1 & 2,2 & 2 & 3,6 \\
Vaginitis aeróbica & 2 & 2,0 & 1 & 2,2 & 1 & 1,8 \\
Total & 101 & 100 & 46 & 100 & 55 & 100 \\
\hline
\end{tabular}

Tabla 3. Frecuencia de infecciones vaginales en relación con la edad de las pacientes. Centro de Salud Familiar de Maipú, Región Metropolitana, Chile

\begin{tabular}{cccc|}
$\begin{array}{c}\text { Grupo etario } \\
\text { (años) }\end{array}$ & $\begin{array}{c}\text { Consultantes } \\
\text { n }\end{array}$ & $\mathbf{n}$ & $\begin{array}{c}\text { Infección vaginal } \\
\text { Frecuencia (\%) }\end{array}$ \\
$15-24$ & 38 & 20 & 52,6 \\
$25-34$ & 35 & 10 & 28,6 \\
$35-54$ & 28 & 17 & 60,7 \\
\hline
\end{tabular}

embarazadas 33,5 años (DE: 10,88), diferencia no significativa (p: 0,5742). El motivo de consulta más frecuente fue control prenatal $(36,6 \%)$, seguido por síntomas de infección vaginal $(32,7 \%)$ y control ginecológico $(17,8 \%)$. En 13 pacientes no se anotó el motivo de consulta.

Se estudió la microbiota vaginal de las 101 mujeres. En 54 (53,5\%) de ellas, la microbiota fue categorizada como normal y en 47 casos $(46,5 \%)$ se detectó una o más infecciones vaginales. No hubo diferencias significativas en la proporción de microbiota normal o infecciones entre gestantes y no gestantes. En la Tabla 2 se observan los resultados microbiológicos totales y agrupados en mujeres embarazadas y en no embarazadas. La VB, seguida por la CVV, fueron las infecciones vaginales más frecuentes en la población general y en las mujeres no embarazadas, mientras que en las mujeres gestantes, la frecuencia de estas infecciones fue similar. Se obtuvieron 13 aislados de Candida en las 12 pacientes con CVV. Nueve de ellos correspondieron a C. albicans $(69,2 \%)$, dos $(15,4 \%)$ a $C$. tropicalis, uno $(7,7 \%)$ a $C$. glabrata y un caso $(7,7 \%)$ a la asociación de $C$. albicans y $C$. glabrata. Se detectaron siete co-infecciones; seis correspondieron a la asociación de CVV y VB y una a la asociación de VB y VA. Las infecciones menos frecuentes fueron tricomoniasis $\mathrm{y}$ VA, con tres y dos casos respectivamente. Los casos de tricomoniasis fueron diagnosticados por RPC (n: 3), cultivo (n: 2) y examen al fresco (n: 1). Cinco muestras fueron inadecuadas para el análisis por RPC.

En la Tabla 3 se muestra la frecuencia de infección vaginal en relación con la edad, agrupada en tres rangos, de las pacientes. Se observó diferencias significativas entre la frecuencia de infección y el grupo etario (p: 0,0251). El grupo con mayor frecuencia de infecciones vaginales correspondió al de 35-44 años. La frecuencia de infección en este grupo fue significativamente mayor a la encontrada en el grupo de 25-34 años (p: 0,020).

La Figura 1 muestra la frecuencia de VB, CVV y co-infecciones en los tres rangos etarios. El grupo de de 15-24 años presentó la mayor frecuencia de co-infecciones vaginales, mientras que las mujeres de 35-54 años presentaron la mayor y menor frecuencia de VB y CVV, respectivamente (p: 0,899).

Treinta y tres mujeres $(32,7 \%)$ notificaron síntomas de infección vaginal: prurito (n: 16), secreción vaginal (n: 12), prurito y leucorrea (n: 1) e indefinidos (n: 4). En 17 de ellas $(51,5 \%)$, el diagnóstico microbiológico fue positivo. Los diagnósticos más frecuentes fueron CVV (n: 6), VB (n: 5) y MI (n: 3). Por otra parte, 68 mujeres no declararon síntomas de infección vaginal y en 30 de ellas (44,1\%), el diagnóstico microbiológico fue positivo. Se analizó la relación entre la declaración de síntomas de infección vulvo-vaginal y el tipo de síntomas y el diagnóstico microbiológico, no observándose asociación significativa. 
En 80 mujeres (79,2\%), se encontraron signos de infección vaginal en el examen genital. Los signos clínicos fueron categorizados como flujo vaginal alterado (leucorrea) en $50(62,5 \%)$ mujeres, inflamación vulvo-vaginal en 22 casos $(27,5 \%)$, leucorrea e inflamación vulvo-vaginal en cuatro pacientes $(5 \%)$ e indicados solamente como positivos en otros cuatro $(5 \%)$ casos. La concordancia general entre signos y diagnóstico microbiológico fue $62 / 101(61,4 \%) \kappa=0,77$, buena. La concordancia en el grupo de gestantes fue $25 / 46(54,3 \%) \kappa=0,83$, muy buena, mientras que en no gestantes fue $37 / 55(67,3 \%) \kappa=0,75$, buena. En la Tabla 4 se relacionan los signos clínicos con los resultados microbiológicos. De los 39 casos en que no hubo concordancia entre signos y diagnóstico microbiológico, en tres pacientes sin signos de infección el diagnóstico microbiológico fue positivo, mientras que en 30 pacientes con leucorrea, cuatro con inflamación vulvovaginal y dos con signos no especificados, el diagnóstico microbiológico fue negativo. La concordancia entre leucorrea y diagnóstico de infección vaginal fue $\kappa=0,297$, débil y entre inflamación vulvo-vaginal y diagnóstico de infección vaginal, $\kappa=0,178$, baja. No hubo diferencias significativas entre la presencia de signos de leucorrea o inflamación vulvo-vaginal y los resultados del diagnóstico microbiológico (p: 0,4208).

\section{Discusión}

Los objetivos de este estudio fueron determinar la prevalencia y tipos de infección vaginal en un CESFAM de la RM para lo cual se incluyeron consecutivamente mujeres con distintos motivos de consulta. Se encontró una alta frecuencia de infecciones vaginales lo que sugiere investigar los factores de riesgo que están determinando esta frecuencia, dado su fuerte conexión con patologías obstétricas, ginecológicas y transmisión de ITS.

La VB fue la infección vaginal más frecuentemente detectada. La VB es una alteración compleja de la microbiota vaginal, en la que los lactobacilos van siendo reemplazados secuencialmente por Gardnerella vaginalis y un número creciente de bacterias anaerobias, siendo Prevotella spp, Peptostreptococcus spp y Mobiluncus spp las especies más frecuentemente cultivadas. La infección produce generalmente supresión de la respuesta inflamatoria local y las portadoras de ella tienen mayor riesgo de desarrollar enfermedad inflamatoria pélvica, PP, endometritis puerperal y de adquirir ITS ${ }^{3-6}$. Su frecuencia varía ampliamente según la población estudiada, lo que se refleja también en estudios efectuados en nuestro medio: adolescentes $31 \%^{20}$, planificación familiar $32 \%{ }^{1,2}$ y $61 \%$ en trabajadoras sexuales ${ }^{21}$. Su prevalencia en mujeres con patología obstétrica y ginecológica es mayor que en la población general, habiendo sido detectada en $35 \%$ de

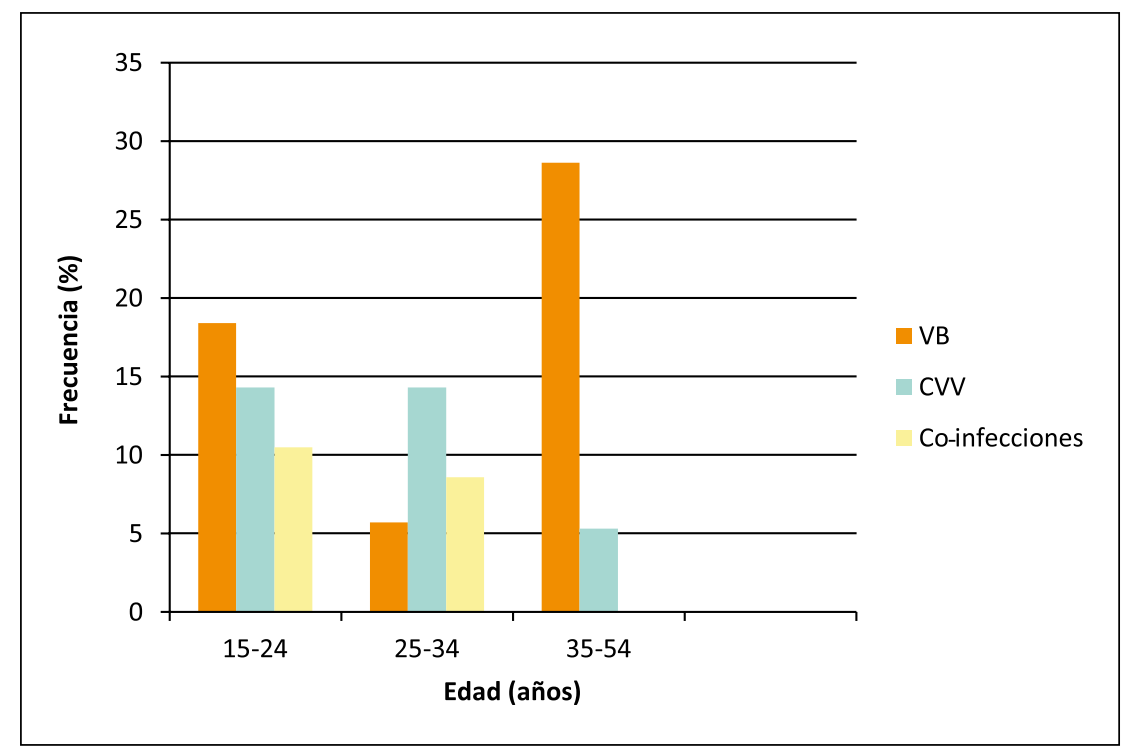

Figura 1. Frecuencia de vaginosis bacteriana (VB), candidiasis vulvo-vaginal (CVV) y co-infecciones en relación con edad de las pacientes.

Tabla 4. Relación entre la presencia de signos de infección vulvo-vaginal y los resultados microbiológicos

\begin{tabular}{|c|c|c|c|c|c|c|c|c|}
\hline \multirow[b]{2}{*}{ Signos (n) } & & \multicolumn{7}{|c|}{ Diagnóstico microbiológico } \\
\hline & & Normal & VB & CVV & $\begin{array}{l}\text { Co-infec- } \\
\text { ciones }\end{array}$ & $\begin{array}{l}\text { Microbiota } \\
\text { intermedia }\end{array}$ & Tricomoniasis & VA \\
\hline Sin signos & $(21)$ & 18 & 2 & 1 & - & - & - & \\
\hline Leucorrea & $(50)$ & 30 & 9 & 3 & 2 & 2 & 3 & 1 \\
\hline Inflamación & $(22)$ & 4 & 5 & 6 & 4 & 2 & - & 1 \\
\hline Mixtos & (4) & - & 1 & 2 & 1 & - & - & \\
\hline No especifica & $\operatorname{dos}(4)$ & 2 & - & - & - & 2 & - & \\
\hline Total & (101) & 54 & 17 & 12 & 7 & 6 & 3 & 2 \\
\hline
\end{tabular}

mujeres con rotura prematura de membranas en el embarazo de pretérmino ${ }^{22}, 43 \%$ de mujeres con parto prematuro con membranas intactas sin causa clínica evidente ${ }^{23}$ y en $50 \%$ de pacientes con enfermedad inflamatoria pélvica aguda $^{24}$. La patogenia de la VB no es bien conocida, pero se han identificado numerosos factores de riesgo. La VB ha sido asociada con contacto con una nueva o múltiples parejas heterosexuales o del mismo género y menor uso de preservativos ${ }^{14,25}$. La prevalencia de VB también se asocia significativamente con estrés psicosocial crónico en mujeres embarazadas y no embarazadas ${ }^{26}$, tabaquismo y hábitos de higiene como el uso de duchas vaginales ${ }^{27}$. El diagnóstico de VB puede efectuarse clínicamente o mediante el test de Nugent y cols., Este último es el más empleado actualmente. 
La CVV constituyó la segunda causa de infección vaginal, aislándose C. albicans en la mayoría de los casos. La CVV es una causa frecuente de infección vaginal en el mundo $0^{4,5,7}$. Es un hallazgo frecuente en mujeres gestantes, pero no existen evidencias que la relacionen con $\mathrm{PP}^{4,22,23}$. No obstante, en pacientes portadoras de DIU no extraído o con cerclaje, está descrito el aislamiento de estos microorganismos desde el líquido amniótico. En estos casos, Candida se asocia con PP y aborto por intermedio de infección intraamniótica y corioamnionitis histológica ${ }^{28,29}$. Al comparar nuestro estándar microbiológico con los signos y síntomas, encontramos a dos pacientes con cultivo de Candida positivo y signos y síntomas de infección vaginal y microscopia negativa, indicando resultados falsos negativos del examen microscópico. Considerando la menor sensibilidad del examen microscópico, el mejor estándar para el diagnóstico de CVV debería considerar la presencia de elementos levaduriformes al examen microscópico y/o cultivo positivo, en una paciente con clínica de infección vaginal.

La baja prevalencia de $T$. vaginalis en este estudio concuerda con la información existente. Las comunicaciones nacionales más recientes informan prevalencias de 2 a $5,7 \%$ en mujeres adolescentes ${ }^{20,30}, 2,4 \%$ en mujeres embarazadas ${ }^{16}$ y $6,8 \%$ en mujeres en atención primaria ${ }^{31}$. A nivel global, la prevalencia de tricomoniasis varía ampliamente, siendo mayor en países pobres en vías de desarrollo, en personas de raza negra, en poblaciones con escaso acceso a la salud y por razones biológicas en la mujer ${ }^{32}$. A diferencia de otras ITS, que tienen mayor prevalencia en adolescentes y mujeres de menor edad, la tricomoniasis se distribuye en todos los grupos etarios ${ }^{33}$. Aunque la tricomoniasis en mujeres es hasta 50\% asintomática, la disponibilidad en nuestro país de atención sanitaria, especialmente control prenatal y disponibilidad de Papanicolaou han favorecido probablemente su baja prevalencia. Trichomonas vaginalis es un factor de riesgo importante para PP, niños de bajo peso de nacimiento y de transmisión de ITS bacterianas y virales ${ }^{34}$. El diagnóstico microbiológico de la tricomoniasis se efectúa generalmente mediante examen al fresco, cuya sensibilidad no supera el $50 \%$. El cultivo en caldo Diamond tiene mayor sensibilidad, pero es muy demandante y el acceso a medios de cultivo comerciales es deficiente, por lo que en la práctica no se emplea en los laboratorios clínicos. El diagnóstico molecular mediante RPC es la técnica de mayor sensibilidad y podría ser de gran utilidad en mujeres en las que hay sospecha clínica, pero el examen microscópico es negativo ${ }^{33}$.

Se encontró una baja prevalencia de VA, siendo éste el primer estudio que comunica VA en nuestro medio. La VA es una infección vaginal cuya descripción clínica y microbiológica es relativamente reciente y por ello su prevalencia es aún poco conocida ${ }^{6,12}$. Es un proceso infeccioso similar a la VB en el sentido de que también se caracteriza por la pérdida del predominio lactobaci$1 \mathrm{lar}^{6,12}$, pero, a diferencia de VB, en su etiología participan bacterias anaerobias facultativas, como Escherichia coli, Streptococcus agalactiae, Staphylococcus aureus y Enterococcus faecalis. Estas bacterias colonizan la mucosa vaginal en forma persistente causando una intensa respuesta inflamatoria vaginal y niveles de citoquinas pro-inflamatorias en el contenido vaginal significativamente superiores a los encontrados en mujeres con $\mathrm{VB}^{6}$. Existen evidencias que la VA se asocia con un riesgo mayor de $\mathrm{PP}$ que $\mathrm{VB}^{6,12}$. El diagnóstico de $\mathrm{VB}$ se efectúa microscópicamente, atendiendo a un sistema de puntajes que considera la proporción de lactobacilos con respecto de otros morfotipos bacterianos, la cantidad de leucocitos, proporción de leucocitos de aspecto tóxico y proporción de células epiteliales parabasales. De acuerdo a este protocolo, el diagnóstico de VA es leve, moderado o severo. La secreción vaginal en VA se caracteriza por ser espesa, de color amarillento y de mal olor ${ }^{12}$. Los signos y síntomas de VA no son específicos y es difícil diagnosticarla sin la ayuda del laboratorio.

Cerca de $7 \%$ de las pacientes presentaron co-infecciones, la mayoría CVV con VB, lo que ha sido descrito ${ }^{11}$. La distinción entre una co-infección y una infección mixta tiene implicancias terapéuticas, pero la mayoría de los estudios no diferencia entre ambas, ya que no existe un estándar microbiológico para su definición. Una coinfección correspondería a la concurrencia de dos infecciones vaginales, sin que se establezca la contribución de cada uno a la alteración de la homeostasis vaginal ${ }^{35}$. Una infección mixta, también denominada vaginitis mixta, corresponde a la concurrencia de dos procesos infecciosos, ambos contribuyendo a la alteración de la homeostasis vaginal y a la producción de signos y síntomas ${ }^{35}$. No es posible del punto de vista microbiológico distinguir entre co-infección y una vaginitis mixta por CVV y VB, ya que el método de Nugent no emplea parámetros clínicos y los síntomas y signos no contribuyeron a distinguirlas. La frecuencia de mujeres con CVV y VB definida por microscopia, varía de 3 a 27\% dependiendo de la población estudiada ${ }^{35}$.

El 6\% de las pacientes presentó microbiota intermedia. El significado clínico de esta categoría de microbiota sigue siendo motivo de controversia entre aquellos que sugieren que es una entidad clínica específica, o aquellos que indican que es parte de un continuum entre microbiota normal y $\mathrm{VB}^{5,6,15}$. No obstante, ha sido descrita como factor de riesgo de aborto y $\mathrm{PP}^{5,6}$. En este estudio, las seis mujeres con MI presentaron signos de infección vaginal. No existen criterios clínicos para su diagnóstico y el diagnóstico se establece mediante criterios microscópicos para los cuales no existe consenso.

El estudio incluyó, por azar, un número similar de 
mujeres gestantes y no gestantes y no se observaron diferencias significativas en la edad ni en la frecuencia de infección vaginal entre ambos grupos. No obstante, al separar la población estudiada en tres grupos etarios y determinar la frecuencia de infección vaginal, se observaron diferencias significativas, siendo la frecuencia de infección vaginal en el grupo de mayor edad significativamente más alta que en el de 25-34 años. Esto podría explicarse por el motivo de consulta. El $50 \%$ de las mujeres del grupo de mayor edad consultó por síntomas de infección, mientras que sólo 7 (20\%) de las mujeres de 25-34 años consultaron por esta razón (p: 0,0163).

No encontramos una buena correlación entre síntomas y diagnóstico microbiológico de infección vaginal, lo que ha sido también demostrado en otros estudios ${ }^{8-10}$. En casi $50 \%$ de las mujeres que declararon síntomas no se encontró una etiología infecciosa. Existen causas no infecciosas de vaginitis, como las alergias de contacto a látex, lubricantes, compresas y las irritaciones producidas por el uso de jabones u otros productos de higiene inadecuados, que podrían explicar parte de la sintomatología, pero no disponemos de esta información. La capacidad de la mujer de discriminar entre lo que es fisiológico y lo anormal también pudo contribuir a la discordancia. Finalmente, el pequeño tamaño de la muestra y la acotada declaración de los síntomas contribuyeron a estos resultados. Sería importante desarrollar un panel de preguntas con un mayor poder de discriminación y que pueda ayudar también a las mujeres en su reconocimiento y descripción, como asimismo indagar en las prácticas de higiene y otros hábitos de las pacientes.

Los signos clínicos tuvieron una mayor capacidad de discriminación que los síntomas y buena concordancia con el diagnóstico de laboratorio, pero la concordancia con infecciones vaginales específicas fue débil a moderada. El diagnóstico microbiológico no incluyó todas las causas de infección vaginal como la lactobacilosis citolítica y las infecciones virales. Whatne y cols., demostraron la presencia de virus herpes simplex en $7 \%$ de mujeres con leucorrea ${ }^{36}$. Los autores también demostraron la contribución de las infecciones cervicales a la presencia de leucorrea e inflamación ${ }^{36}$. Los factores genéticos son determinantes en la respuesta inmune a antígenos microbianos y, ante una exposición, algunas personas pueden montar una reacción inflamatoria, mientras que en otras no se produce tal respuesta ${ }^{37}$. Asimismo, la respuesta inmunológica de un hospedero también varía según su estado de inmunocompetencia y del estrés psicosocial ${ }^{37}$.
Finalmente, la sensibilidad del diagnóstico microbiológico depende de los estándares de diagnóstico y, como se comentara anteriormente, detectamos algunos resultados falsamente negativos de CVV. Por todo lo señalado, no es posible comparar la exactitud entre el diagnóstico microbiológico y el diagnóstico clínico, solamente su concordancia.

En conclusión, se encontró alta frecuencia de infecciones vaginales. Es necesario mejorar las definiciones y criterios de diagnóstico microbiológico de las coinfecciones y microbiota intermedia, para poder ser diagnosticadas en la práctica clínica. Se recomienda emplear cuestionarios más descriptivos para mejorar la utilidad del examen clínico.

\section{Resumen}

Introducción: Las infección vaginales constituyen un motivo frecuente de consulta, pero su prevalencia y etiología varían en distintas poblaciones. Objetivos: Determinar la prevalencia y tipos de infección vaginal en mujeres atendidas en un centro de salud familiar de la Región Metropolitana. Métodos: El diagnóstico microbiológico fue efectuado mediante examen microscópico al fresco y tinción de Gram y para tricomoniasis examen al fresco, cultivo y reacción de la polimerasa en cadena. Resultados: Se incluyeron 101 mujeres de 15-54 años, no seleccionadas por signos ó síntomas, 46 de ellas embarazadas. En 47 mujeres $(46,5 \%)$ se diagnosticaron infecciones vaginales. Se observó asociación entre edad y frecuencia de infección vaginal. La proporción de infecciones entre gestantes y no gestantes fue similar. Las infecciones más frecuentes fueron vaginosis bacteriana $(16,8 \%)$, candidiasis vulvo-vaginal $(11,9 \%)$ y co-infecciones $(6,9 \%)$. Se observó $5,9 \%$ casos de microbiota intermedia, 3\% de tricomoniasis y $2 \%$ de vaginitis aeróbica. Los síntomas de infección vaginal tuvieron mala concordancia con los hallazgos microbiológicos. A su vez, los signos físicos tuvieron buena concordancia con la existencia de infección, pero escasa a moderada concordancia con una etiología específica. Conclusiones: Se encontró alta frecuencia de infecciones vaginales. Es necesario mejorar las definiciones y criterios de diagnóstico microbiológico de las co-infecciones y microbiota intermedia, para ser diagnosticadas en la práctica clínica. Se recomienda emplear cuestionarios más descriptivos para mejorar la utilidad del examen clínico. 


\section{Referencias bibliográficas}

1.- Navarrete P, Domínguez M, Castro E, Zemelman R. Evaluación de los criterios de Nugent y Amsel para el diagnóstico de vaginosis bacteriana. Rev Med Chile 2000; 128: 767-71.

2.- Lillo G E, Lizama I S, Medel C J, Martínez M A. Diagnóstico de vaginosis bacteriana en un consultorio de planificación familiar de la Región Metropolitana, Chile. Rev Chilena Infectol 2010; 27: 199-203.

3.- Hay P E, Lamont R F, Taylor-Robinson D, Morgan D J, Ison C, Pearson J. Abnormal bacterial colonization of the genital tract and subsequent preterm delivery and late miscarriage. Br Med J 1994; 308: 295-8.

4.- Ovalle A, Martínez M A. Infección genital. Eghon Guzmán, Editor. Selección de temas de Ginecoobstetricia Tomo II. 1 ed. Santiago de Chile: Publiimpacto; 2007, p.875-923.

5.- Donati L, Di Vico A, Nucci M, Quagliozzi L, Spagnuolo T, Labianca A, et al. Vaginal microbial flora and outcome of pregnancy. Arch Gynecol Obstet 2010; 28: 589-600.

6.- Donders GG, Van Calsteren K, Bellen G, Reybrouck R, Van den Bosch T, Riphagen I et al. Predictive value for preterm birth of abnormal vaginal flora, bacterial vaginosis and aerobic vaginitis during the first trimester of pregnancy. BJOG 2009; 116: 1315-24.

7.- Hainer B L, Gibson M V. Vaginitis: diagnosis and treatment. Am Fam Physician 2011; 83: 807-15.

8.- Anderson M R, Klink K. Cohrssen A. Evaluation of vaginal complaints. JAMA 2004; 291: 1368-79.

9.- Landers D V, Wiesenfeld H C, Heine R P, Krohn M A, Hillier S L. Predictive value of the clinical diagnosis of lower genital tract infection in women. Am J Obstet Gynecol 2004; 190: 1004-10.

10.- Schwiertz A, Taras D, Rusch K, Rusch V. Throwing the dice for the diagnosis of vaginal complaints?. Ann Clin Microbiol Antimicrob 2006; 5: 4. [PMC free article]

11.- Rivers C A, Adaramola O O, Schwebke J R. Prevalence of bacterial vaginosis and vulvovaginal candidiasis mixed infection in a southeastern American STS clinic. Sex Transm Dis 2011; 38: 672-4.

12.- Donders G G, Bellen G, Rezeberga D. Aerobic vaginitis in pregnancy. BJOG 2011; 118: $1163-70$

13.- Rylander E, Berglund A-L, Krassny C, Petrini B. Vulvovaginal candida in a young sexually active population: prevalence and association with oro-genital sex and frequent pain at intercourse. Sex Transm Infect 2004; 80: 54-7.
14.- Fethers K A, Fairley C K, Hocking J S, Gurrin L C, Bradshaw C S. Sexual risk factors and bacterial vaginosis: A systematic review and meta-analysis. Clin Infect Dis 2008; 47: 1426-35.

15.- Nugent R, Krohn M A, Hillier S L. Reliability of diagnosing bacterial vaginosis is improved by a standardized method of Gram stain interpretation. J Clin Microbiol 1991; 29: 297-301.

16.- Ovalle A, Martínez M A, de la Fuente F, Falcon N, Feliú F, Fuentealba F, et al. Prevalencia de infecciones de transmisión sexual en mujeres embarazadas atendidas en un hospital público de Chile Rev Chilena Infectol 2012; 29: 521-4.

17.- Gatica J L, Goic I, Martínez MA, Reid I, Céspedes P, Arias M C, et al. Utilidad del Agar CROMOCANDIDA para el diagnóstico diferencial de Candida spp. aisladas de muestras vaginales. Rev Chil Obstet Ginecol 2002; 67 : 300-04.

18.- Kengne P, Veas F, Vidal N, Rey J L, Cuny G. Trichomonas vaginalis: repeated DNA target for highly sensitive and specific polymerase chain reaction diagnosis. Cell Mol Biol 1994; 40: 819-31.

19.- Chomczynski P, Mackey K, Drews R, Wilfinger W. DNAzol: a reagent for the rapid isolation of genomic DNA. Biotechniques 1997; 22: 550-3.

20.- Martínez M A, Barría A, Meneses R, Oyarzún P, Sandoval J. Vulvovaginitis en la adolescencia. Estudio etiológico. Rev Chil Obstet Ginecol 2003; 68: 499-502.

21.- Venegas G, Boggiano G, Castro E. Prevalencia de vaginosis bacteriana en trabajadoras sexuales chilenas. Rev Panam Salud Pública 2011; 30: 46-50.

22.- Ovalle A, Martínez MA, Gómez R, Sáez J, Menares I, Aspillaga C, et al. Parto prematuro con membranas intactas: microbiología del líquido amniótico y tracto genital inferior y su relación con los resultados materno neonatales. Rev Med Chile 2000; 128: 985-95.

23.- Ovalle A, Gómez R, Martínez MA, Kakarieka E, Fuentes A, Aspillaga C, et al. Invasión microbiana de la cavidad amniotica en la rotura de membranas de pretérmino. Resultados materno-neonatales y patología placentaria según microorganismo aislado. Rev Med Chile 2005; 133: 51-61.

24.- Ovalle A, Martínez M A, Casals A, Yuhaniak R, Giglio M S. Estudio clínico y microbiológico de la enfermedad inflamatoria pélvica aguda. Rev Chil Obstet Ginecol 1993; 58:103-12.

25.- Smart S, Singal A, Mindel A. Social and sexual risk factors for bacterial vaginosis. Sex Transm Infect 2004; 80: 58-62.
26.- Nansel T R, Riggs M A, Yu K F, Andrews W W, Schwebke J R, Klebanoff M A. The association of psychosocial stress and bacterial vaginosis in a longitudinal cohort. Am J Obstet Gynecol. 2006; 194: 381-6.

27.- Larsson P G, Fåhraeus L, Carlsson B, Jakobsson T, Forsum U. Predisposing factors for bacterial vaginosis, treatment efficacy and pregnancy outcome among term deliveries; results from a preterm delivery study. BMC Womens Health 2007; 7:20. Doi: 10.1186/14726874-7-20.

28.- Cotch M F, Hillier S L, Gibbs R S, Eschenbach D A. Epidemiology and outcomes associated with moderate to heavy Candida colonization during pregnancy. Vaginal Infections and Prematurity Study Group. Am J Obstet Gynecol 1998; 178: 374-80.

29.- Roqué H, Abdelhak Y, Young B K. Intra amniotic candidiasis. Case report and metaanalysis of 54 cases. J Perinat Med 1999; 27 : 253-62.

30.- Sagua H, Chiang V, Varela H, Neira I, Bugueño $\mathrm{M}$, Contreras $\mathrm{C}$, et al. Trichomoniasis en adolescentes embarazadas de Antofagasta, Chile. Parasitol Día 1999; 23: 9-14.

31.- Neira P, Correa A, Muñoz N, Tardío MT, Carabelli M. Frecuencia de infección por Trichomonas vaginalis en atención primaria de salud. Rev Chil Obstet Ginecol 2005; 70: 147-51.

32.- Bowden F J, Garnett G P. Trichomonas vaginalis epidemiology: parameterising and analysing a model of treatment interventions. Sex Transm Infect 2000; 76: 248-56.

33.- Bachmann L H, Hobbs M M, Seña A C, Sobel J D, Schwebke J R, Krieger J N, et al. Trichomonas vaginalis genital infections: progress and challenges. Clin Infect Dis 2011; 53 (Suppl3): S160-72.

34.- Cotch M F, Pastorek J G, Nugent R P, Hillier S L, Gibbs RS, Martin D H, et al. Trichomonas vaginalis associated with low birth weight and preterm delivery. The vaginal infections and prematurity study group. Sex Trans Dis 1997; 24: 353-60.

35.- Sobel J D, Subramanian C, Foxman B, Fairfax M, Gygax S E. Mixed vaginitismore than coinfection and with therapeutic implications. Curr Infect Dis Rep 2013; 15 : 104-8.

36.- Whatne B, Holst E, Hovelius B, Mårdh P A. Vaginal discharge-comparison of clinical, laboratory and microbiological findings. Acta Obstet Gynecol Scand 1994; 73: 802-8.

37.- Osborne N G, Grubin L, Pratson L. Vaginitis in sexually active women: relationship to nine sexually transmitted organisms. Am J Obstet Gynecol 1982; 962-7. 